\title{
Mechanisms of layer growth in microwave-PECVD silan plasmas - Experiment and simulation
}

\author{
E. Ramisch ${ }^{a}$, A. Mutzke ${ }^{b}$, R. Schneider ${ }^{c}$, U. Stroth ${ }^{d}$ \\ ${ }^{a}$ Institut für Plasmaforschung, Universität Stuttgart, Pfaffenwaldring 31, 70569 \\ Stuttgart, Germany \\ ${ }^{b}$ Max-Planck-Institut für Plasmaphysik, EURATOM Association, Wendelsteinstr. \\ 1, 17491 Greifswald, Germany \\ ${ }^{c}$ Institut of Physics, Ernst-Morits-Arndt University, Felix-Haussdorffstr. 1, 17491 \\ Greifswald, Germany \\ ${ }^{d}$ Max-Planck-Institut für Plasmaphysik, EURATOM Association, Boltzmannstr 2, \\ 85748 Graching, Germany
}

\begin{abstract}
For the specific manipulation of barrier-layer properties, a detailed analysis of the layer-growth mechanisms in microwave-PECVD was carried out for Si-wafers with a trench structure as "model cavities" in the $\mu m$ range. The deposition of a-Si:H (hydrogenated amorphous silicon) layers in pure monosilane plasmas was used as model system to compare experimental results and simulations using the 2D binary collision code SDTrimSP-2D, which showed very good agreement with the experiment. Without bias the layer tends to close above the cavities from both sides, but cracks remain at the closure positions. By biasing the substrate a smoothing of the layer edges above the cavities occurred. Thus, the cavities remained open for a longer time and a more homogeneous coating of the notches is obtained.
\end{abstract}

Key words: Atoms sputtering, atom, molecule, ion impact, applications of Monte

Preprint submitted to Elsevier 
Carlo method, Numerical methods, film deposition,

PACS: 34.50, 79.20.R, 02.50.U, 02.60, 81.15.C,

\section{Introduction}

The demand for environment-friendly energy sources increases. In this context, the focus is set mainly on the development of wind power and solar energy with competitive production costs. Above all, this is a problem for solar cells, which, today, are mainly fabricated out of crystalline silicon and, therefore, are in competition with semiconductor industry. Hence, the development of solar cells based on alternative materials like e.g. copper-indium-gallium-diselenide (CIGS) is of great interest. Because of the lower layer thickness needed for this material, these solar cells can be fabricated on flexible substrates like metal foils. This possibility offers a broader spectrum of applications. For reaching low production costs, the applicability of unpolished steel foil, which exhibits scratches on the $\mu \mathrm{m}$ scale, is investigated as substrate for the solar cells in this work. The use of any metal as substrate requires a barrier layer between the substrate and the solar cells to prevent short-circuits between the separate cells of a solar module and to prevent the diffusion of undesired substrate elements into the solar cells. To produce barrier coatings, silicon oxide $\left(\mathrm{SiO}_{\mathrm{x}}\right)$ and silicon nitride $\left(\mathrm{SiN}_{\mathrm{y}}\right)$ were deposited in a microwave-PECVD process in the Plasmodul setup. High-energetic ions impinging on the layer surface lead to a layer smoothing and melting, especially at positions of indentations in the substrate surface. Hence, the barrier properties of the coating are improved.

The aim of this work is a better understanding of the effects of the impinge of highenergetic ions on the layer surface during the deposition. For good barrier properties, the development of pinholes in the coating has to be avoided. These defects mainly appear at positions of notches on the substrate surface. Hence, the layer growth at such positions had to be analyzed to explain the formation of pinholes and find solutions for avoiding them. This was done on the basis of model substrates with a well-defined trench surface structure in the $\mu \mathrm{m}$ range serving as "model cavities" for biased and unbiased cases. 
The processes, which lead to different profiles of the biased and unbiased layers at positions of indentations on the substrate surface, were determined by detailed analysis of the local layer-forming mechanisms like the loss of particles, sputtering and redeposition at the film surface. In particular, the time-dependent process of the layer closure above the cavities is of special interest. These studies were carried out by means of numerical simulations using the Monte-Carlo Code SDTrimSP-2D [1]. Compared to former simulation codes, this one offers the possibility of including the impacting high-energetic ions in the process on the substrate surface and, therewith, indirectly the effect of the substrate bias. Since chemical reactions are not implemented in the SDTrimSP-2D code, simulations of a-Si:H (hydrogenated amorphous silicon) layer depositions were carried out in a pure monosilane plasma acting as model system, in which chemical reactions can be neglected.

In the following, the experimental set-up and the Monte-Carlo Code SDTrimSP-2D are introduced. The input parameters will be determined, the simulation results will be compared with the experiment and the particular film-forming processes, especially in the case of the applied substrate bias, will be discussed. Finally, the results will be summarized.

\section{Experiment}

The Plasmodul [2] is a low-pressure microwave plasma device. To guarantee its modular handling, each task is integrated in its own module and, hence, the different parts can be substituted easily. This ensures a quick adaption of the setup to the particular demand of the plasma process. In the present setup, the whole device has a cylindrical basic form with an outer diameter of $35 \mathrm{~cm}$ and a height of $52 \mathrm{~cm}$. The eight separate modules are aluminum rings with a wall thickness of $2.5 \mathrm{~cm}$. The separate modules sealed with O-rings are placed on each other and fixed by a fastening. The main parts are the two separate gas inlets, the plasma source and the reaction chamber. With the top-arranged exhaustion, they build an upstream plasma device. The advantage of such a configuration is the possibility of an overhead deposition, which prevents a contamination of the substrate with down-falling tinsels. A schematic view of the device with the particular modular 
parts is illustrated in Fig. 1.

The reactor is completed at the bottom and at the top with a plane cover plate. The plasma source, which consists of an array of four Duo-Plasmalines [3], is embedded into the two gas inlets. With the frequency of $2.45 \mathrm{GHz}$ fed in the Duo-Plasmalines, this leads to a remote microwave plasma deposition process. The standard setting of the supplied microwave power for the presented coatings is $2 \times 600 \mathrm{~W}$. In this configuration possible carrier gases are injected by an electronic mass-flow controller through the lower gas inlet, which is subdivided into four upwardly oriented tubes in order to ensure a uniform gas flow through the whole setup area. The precursor gas, in case of the presented work monosilane, is fed remotely in the upper gas inlet by an electronic mass flow controller, which is split symmetrically into 16 gas outlets leading to a homogeneous deposition on the substrate.

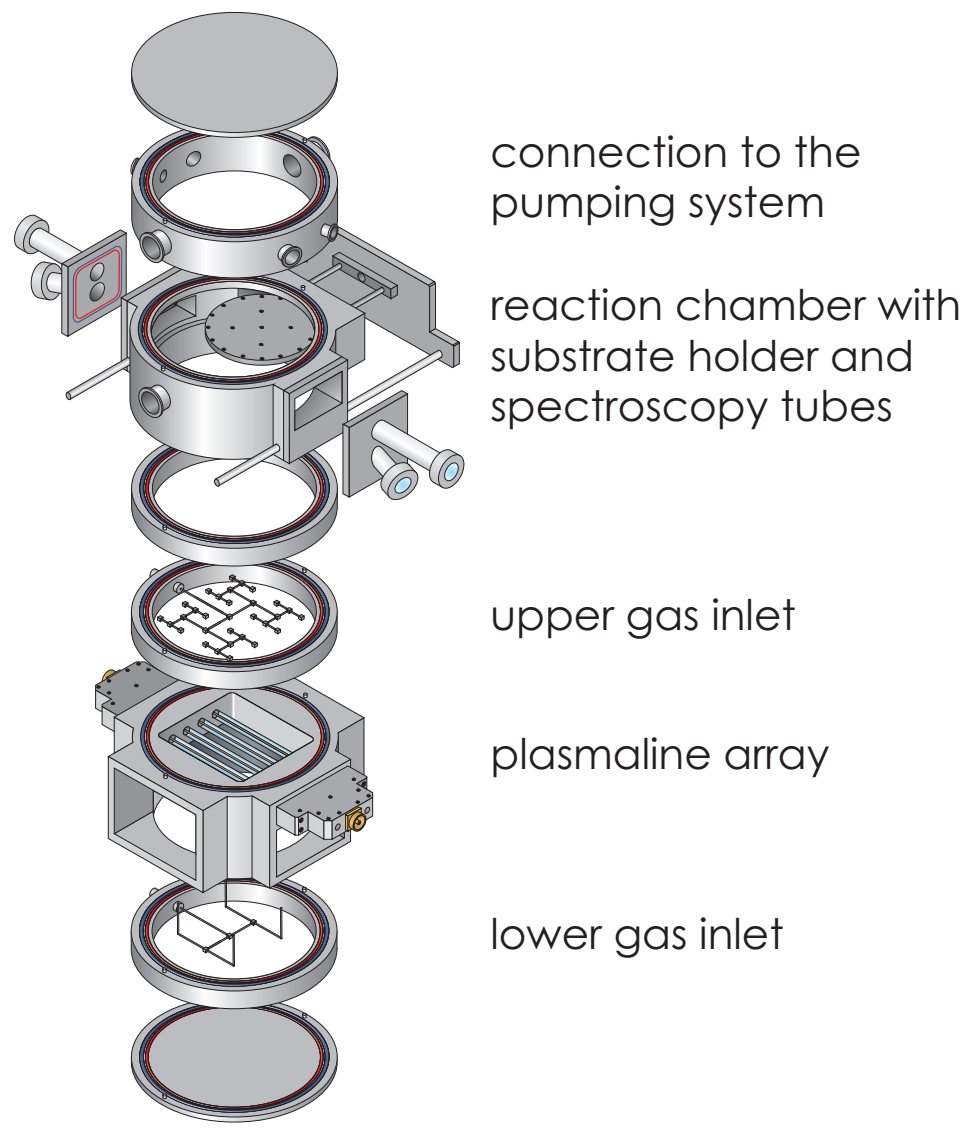

Fig. 1. Schematic view of the used configuration of the Plasmodul, from [4].

Above the upper gas inlet, the reaction chamber is installed with a separating ring acting 
as a distance piece between the reaction chamber and the module of the upper gas inlet. The main component of the reaction chamber is the substrate holder, at which the samples are fixed overhead. The distance between the substrate holder and the plasmaline array is $20 \mathrm{~cm}$. A feature in this work is the possibility of applying a bias voltage at the substrate holder. It can be biased by a bipolarly pulsed power supply (the output is an asymmetric bipolar rectangular pulse), to reach an optimized efficiency of the bias process: During an ideal rectangular pulse, there is no start-up and run-out phase like for a sinusoidal pulse, because the maximum value is reached instantaneously after switching the polarization. Hence, the start-up time after each change of polarization can be omitted. The asymmetry and bipolarity of the pulse both take the different ion and electron mobility in the plasma into account. The bias frequency is chosen in such a manner that it is less than the ion-plasma frequency. Thus, the ions react instantaneously to a change of the substrate bias and, therefore, the positive ions can be accelerated up to energies determined by the negative amplitude of the applied voltage. Any possible negative ions would be attracted during the positive pulse, which is always $+37 \mathrm{~V}$ due to the constant positive output of the pulser. The negative pulse is superimposed, which is adjustable by the following parameters:

The pulse can be regulated by three parameters: the voltage $U$ (absolute averaged value of the signal), the pulse width $p_{w}$ (duration of the positive pulse) and the frequency $f$. The voltage $U$ can be adjusted up to $500 \mathrm{~V}$. The asymmetry of the positive and negative pulse is adjusted by the pulse width between $400 \mathrm{~ns}$ and $40 \%$ of the period length of the positive pulse. Hence, it is dependent on the adjusted frequency, which can be varied between 50 and $250 \mathrm{kHz}$. The bias voltage is applied to the substrate holder, which represents an additional passive electrode in the microwave plasma (in the unbiased case), with respect to the grounded enclosure of the device. This leads to a generation of a low-frequency asymmetric capacitive plasma discharge by the applied bias voltage, which superimposes the microwave generated plasma. Hence, the ion flux can be regulated by the parameters of the microwave discharge, whereas the ion energy is manipulated independently by the bias parameters and therefore by the capacitive discharge. Additionally, the substrate 
holder contains a mica heating plate and, hence, its temperature can be regulated up to $600{ }^{\circ} \mathrm{C}$ to guarantee a constant, but variable substrate temperature during the deposition process. For most of the coatings within this work, it was kept constant at $T=250$. When applying the bias on the substrate holder, however it cannot be kept constant. The temperature can increase up to $350^{\circ} \mathrm{C}$ likely due to the impinge of high-energetic ions. The temperature is controlled by a temperature sensor, which is inserted by a squeezing lead-through.

The last module ring below the cover plate of the device contains the pumping connection, which is split into two inlets. They are installed symmetrically above the substrate holder to guarantee a homogeneous gas flow through the whole device. The pumping system consists of a rotary and a turbo molecular pump with an end pressure of about $10^{-3} \mathrm{mbar}$.

\section{Monte-Carlo code for analyzing ion-solid interactions}

The Monte-Carlo code SDTrimSP-2D is the two-dimensional extension of the previous one-dimensional version SDTrimSP [5]. Both Monte-Carlo Codes are a very valuable tool for analyzing ion-solid interactions like implantation, backscattering, transmission, sputtering and composition changes of the ion-bombarded amorphous solid. They are mainly used in fusion research to calculate erosion at the reactor wall or the divertor [6]. With the two-dimensional version the influence of a surface structure on the interaction of the incoming particles and the target compounds can be determined.

The beginning of investigations of the layer growth on non-planar substrate surfaces is found at the end of the 1960's. It has its origin in semiconductor industry, where the coating of conduction paths on electrical components is of great interest. The size of these steps are in the $\mu \mathrm{m}$ range. In Ref. [7-9], the step coverage of such trenches is calculated analytically by taking into account the geometric configuration of the substrate surface and the incoming particles in a sputter process. The systems are characterized by screening effects of the step edges and - with increasing layer thickness - self-shadowing of the 
coating. In these first calculations, the specific properties of the different species of the layer-forming particles were not regarded. An extension to this approach is the distinction of the various film-forming particles. By an inclusion of their sticking probability, this problem necessitates a numerical solution, which was treated in several works in the 1970's and 1980's [10-14]. The used simulation algorithm is the Monte-Carlo method in all cases. These analyses calculate the step coverage in dependence of some special process parameters: the substrate temperature (included indirectly by the sticking coefficient), the kind of deposited material as well as the flux and the direction of the incoming particles.

The code SDTrimSP-2D takes all mentioned effects into account, but includes several add-ons compared to previous simulation codes. It calculates the interaction between the incoming particles and the bombarded target by the binary collision approximation [15]. This offers the possibility to analyze the influence of high-energetic ions bombarding the growing layer surface on the film-forming mechanisms. This ion bombardment occurs in the case of an applied substrate bias during the deposition process.

In the utilized version of the simulation code, chemical reactions on the film surface are not considered. Hence, a simple model system has to be regarded, in which these reactions can be neglected. Taking the deposition of silicon nitride as starting point, the simplest gas system, for which chemical reactions do not play an essential role with respect to the deposition, is a pure monosilane plasma. Therefore, the deposition of hydrogenated amorphous silicon layers is considered. In the present plasma, the main film-forming particles are $\mathrm{SiH}_{3}$ radicals. In the case of an applied substrate bias, the impinging $\mathrm{Si}_{2} \mathrm{H}_{4}^{+}$ions have to be taken into account additionally. Because both species are generated in the plasma, no chemical surface reactions occur in this system.

Essentially, SDTrimSP-2D is a 2-D extension of SDTrimSP [5], which, in turn, is a generalized version of the TRIDYN program [16]. It can be run in static or dynamic mode (SD) on sequential or parallel systems (SP). SDTrimSP-2D uses a 2-D mesh to represent the surface morphology, the first dimension is the direction perpendicular to the macroscopic surface plane, and the second is in a direction parallel to that plane. This representation is 
sufficient to simulate the ion bombardment of surfaces with 2D micro-structure extended into the 3rd dimension. It shares the same physical model of ion-surface interactions with other codes of the TRIM family. However, the resolution of a second dimension requires a 2-D domain with separate cells. The code follows the density changes in the target material due to projectile and recoil particles coming to rest after a complete slowing-down at the end of their trajectories. In SDTrimSP/TRIDYN, this is done by a 1-D relaxation of the cells. Each trajectory creates a mass flux in the cells it passes. These fluxes can act as sink or source terms for the particle densities. To ensure particle conservation within the numerical setup, which uses a 1D grid of cells in which each cell has a constant volume density according to the material, volume changes of the $1 \mathrm{D}$ cells (expansion or contraction perpendicular to the surface) are used to represent changes to the number of particles in a cell. In SDTrimSP-2D, this procedure has been extended to 2D, subject to the requirement that all volume changes applied are divergence free. This reflects particle conservation in the projectile-target system expressed by volume changes. For each cell, the resulting mass fluxes are taken to be anisotropic by introducing the anisotropy coefficient of the volume relaxation. The transport into the direction of the surface is greater at into the target. In the simulations presented here an anisotropy coefficient of 0.5 was used. The relaxation process is done in several iterations until the divergence of the mass fluxes (transfer of particles between cells) become zero and steady-state conditions without internal tension are obtained. From this steady-state, divergence-free solution the volume changes are applied. In addition, splitting and annihilation of cells was introduced in SDTrimSP-2D, according to a maximum and minimum number of atoms, to be able to represent creation of holes or strong deposition. Additional diagnostics are used to analyze the results of the numerical computations and are able to provide local values of the sputtering and redeposition yields. 


\section{Input parameters}

To simulate the specific layer growth with the code SDTrimSP-2D, the corresponding input parameters of this deposition process have to be determined. These are the plasma parameters in the Plasmodul as well as the coating parameters obtained by characterizing the deposited films. Because of different coatings deposited with and without substrate bias, the parameters must be defined for each case separately. The effect of the $\mathrm{Si}_{2} \mathrm{H}_{4}^{+}$ ions, influencing the layer growth in the case of an accelerating potential in front of the target, have to be taken into account in the case of an applied substrate bias. Hence, the ions and the $\mathrm{SiH}_{3}$ radicals must be treated independently.

An overview of the input parameters is given in Tab. 1. How they are estimated is described in the following.

\begin{tabular}{|l|cc|}
\hline \multicolumn{3}{|c|}{ impinging particle } \\
\hline parameter & $\mathrm{SiH}_{3}$ radicals & $\mathrm{Si}_{2} \mathrm{H}_{4}^{+}$ions \\
\hline impact energy $[\mathrm{eV}]$ & 1 & 1500 \\
polar impact angle & $0 . .80^{\circ}$ (uniformly distributed) & $0{ }^{\circ}$ \\
particle flux $\left[\mathrm{m}^{-2} \mathrm{~s}^{-1}\right]$ & $1.3 \cdot 10^{20}$ & $6.3 \cdot 10^{18}$ \\
sticking coefficient & 0.25 & 0.25 \\
\hline \hline & coating & biased \\
\hline parameter & $\mathrm{unbiased}$ & 11.8 \\
\hline H content of coating $[\mathrm{at} \%]$ & 16 & 1.67 \\
atomic density of coating $\left[\mathrm{g} / \mathrm{cm}^{3}\right]$ & 1.67 & $2.25[17-19]$ \\
surface binding energy of $\mathrm{Si}[\mathrm{eV}]$ & 2.25 & 0 \\
surface binding energy of $\mathrm{H}[\mathrm{eV}]$ & 0 & 5 \\
cell dimensions $[\mathrm{nm}]$ & 5 & \\
\hline
\end{tabular}

Table 1

Overview of the input parameters of the code SDTrimSP-2D in the case of an unbiased (only $\mathrm{SiH}_{3}$ radicals) and a biased $\left(\mathrm{SiH}_{3}\right.$ radicals and $\mathrm{Si}_{2} \mathrm{H}_{4}^{+}$ions $)$target. 


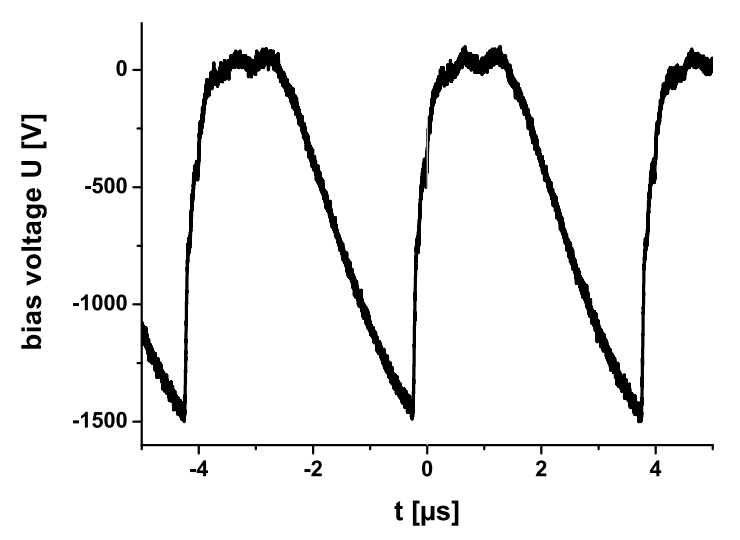

Fig. 2. Voltage characteristics of the applied substrate bias in the monosilane plasma

- Impact energy: The impact energy of the $\mathrm{SiH}_{3}$ radicals on the target is the thermal energy in each case $(1 \mathrm{eV})$, because the flow of neutrals cannot be influenced by an external electric field. Without any substrate bias, the energy of the ions is the thermal one as well. Thus, the energy input of the ions on the layer surface is insignificant and can be neglected. With substrate bias, their energy is determined on the basis of the real voltage characteristics (measured at the standard bias parameters) shown in Fig. 2. Because the ions remain much shorter than one cycle in the sheath region, they can absorb a maximum energy of $1500 \mathrm{eV}$ by crossing the plasma sheath. For simplification, this value is kept constant.

- Sticking coefficient: The sticking coefficient of the $\mathrm{SiH}_{3}$ radicals at an a-Si:H surface is found in literature. It is temperature dependent. In Ref. [20], $s=0.1$ for $T<350{ }^{\circ} \mathrm{C}$ and above this temperature $s=0.25$ is given. In other works, the threshold for a change in the sticking coefficient is given in the range of 250 to $400{ }^{\circ} \mathrm{C}[21-23]$. Here, the sticking coefficient is set to 0.25 for the standard substrate temperature of 250 to $300{ }^{\circ} \mathrm{C}$.

- Target material: The target consists of pure silicon at the beginning of the deposition process. However, the deposited coating includes hydrogen as well. The hydrogen content of the deposited silicon film was measured by the effusion method [24]. An $\mathrm{H}$ content of $11.8 \%$ and $16 \%$ was measured with and without substrate bias, respectively.

- Target density: The density of the deposited layers was determined by measuring the 
mass of the substrate before and after the deposition. It is given by the ratio of the mass difference and the volume of the deposited film.

\section{Comparison of simulation and experiment}

In a first step, the growth of coatings deposited without any substrate bias were simulated. In this case, only thermal processes occur and the flux of $\mathrm{SiH}_{3}$ radicals was varied until a best fit between simulation and experiment was obtained. The flux of the radicals is assumed to be equal in the case of an applied substrate bias. The flux of $\mathrm{Si}_{2} \mathrm{H}_{4}^{+}$ions is some percent of $\phi_{\mathrm{SiH}_{3}}$ [25]. In this work, best agreement of simulations and experiments was obtained for $\phi_{\mathrm{Si}_{2} \mathrm{H}_{4}}=0.05 \phi_{\mathrm{SiH}_{3}}$. The fitting of the flux was done taking as a reference the situation after $30 \mathrm{~min}$ and comparing the simulation results for different fluences for the smooth surface parts of the deposited layer. The fluence which gave the best fit of this layer thickness was chosen. Using this fluence and the known time of $30 \mathrm{~min}$ the flux can be calculated. Combining then the known flux one can calculate for all deposition times the fluence step, which is the primary input for the simulation. This calibration procedure gave very good agreement for all deposition times and reproduced not only the devolpment of the layer thickness, but also the shape of the deposition at the side walls.

Two different aspect ratios of the model substrate are regarded: $a=3$ and $a=1$, describing a relatively small and a wide indentation in the substrate surface, respectively. Figure 3 shows the temporal evolution of the layer forming on these substrates without any substrate bias. A deposition time of 15, 30 and 60 min was chosen. After simulated 60 min of the deposition, the layer is just closed over the smaller cavities. The shape of the $\mathrm{Si}$ wafer before the deposition is depicted as the state at the time $t=0 \mathrm{~min}$. In Fig. 3, the simulation results are plotted over the SEM pictures of the corresponding experimental coatings. A very good agreement of the simulated and measured layer growth is observed in the case of both aspect ratios. The simulation shows the decreasing film thickness alongside the flanks of the bars similar to the real layer as well as the spherical layer growth on the top. The profiles of the coating at the bottom of the trench agrees, 

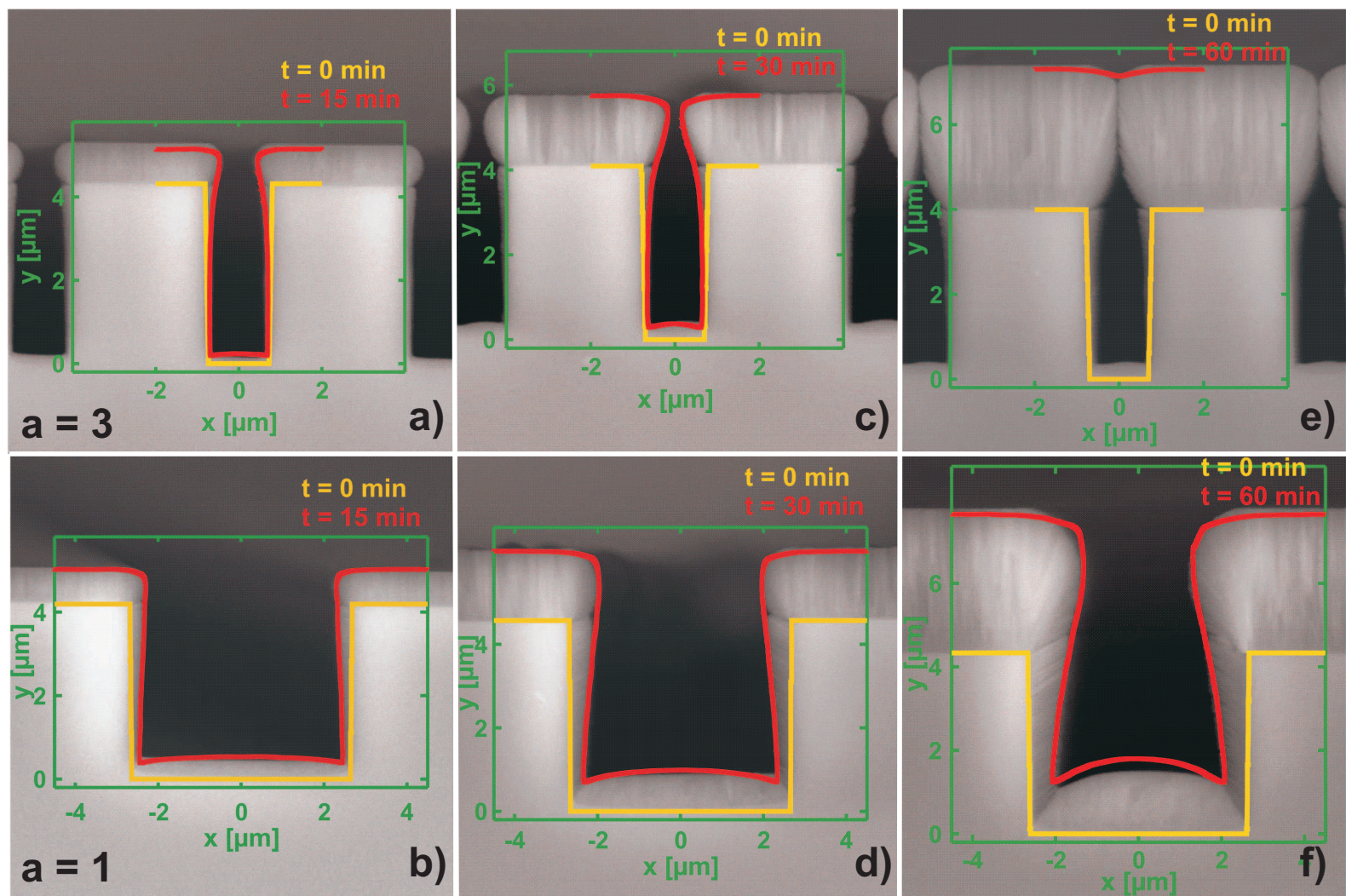

Fig. 3. Comparison of simulated and measured layer growth, deposited without substrate bias onto indentations in the substrate surface. The aspect ratio of the two investigated trenches is $a=3$ and $a=1$. The shape of the original substrate cavity is depicted as the line at $t=0 \mathrm{~min}$. The reflection of incoming particles at the coating surface turns out to be zero at all time steps.

too. In Fig. 3e, the only restriction of the simulation code becomes apparent: If the layer is closed over the indentation the developed cavity is not reproduced, because the code SDTrimSP-2D only computes surface effects. This implies that a crack, which can separate the two film spheres on the enhancements, is not illustrated as well. However, the surface of the coating is reproduced very well. Because of the agreement of the simulations with the experiment during the whole process for different substrate geometries, the choice of the input parameters can be regarded as correct.

Similar investigations were carried out for layers deposited with substrate bias.

The results of the simulation are shown in Fig. 4 in the same representation as in Fig. 3. As for the unbiased coatings, the growth process is well described by the simulation. The sputter effects induced by the energetic ions as observed mainly at the upper edges of 

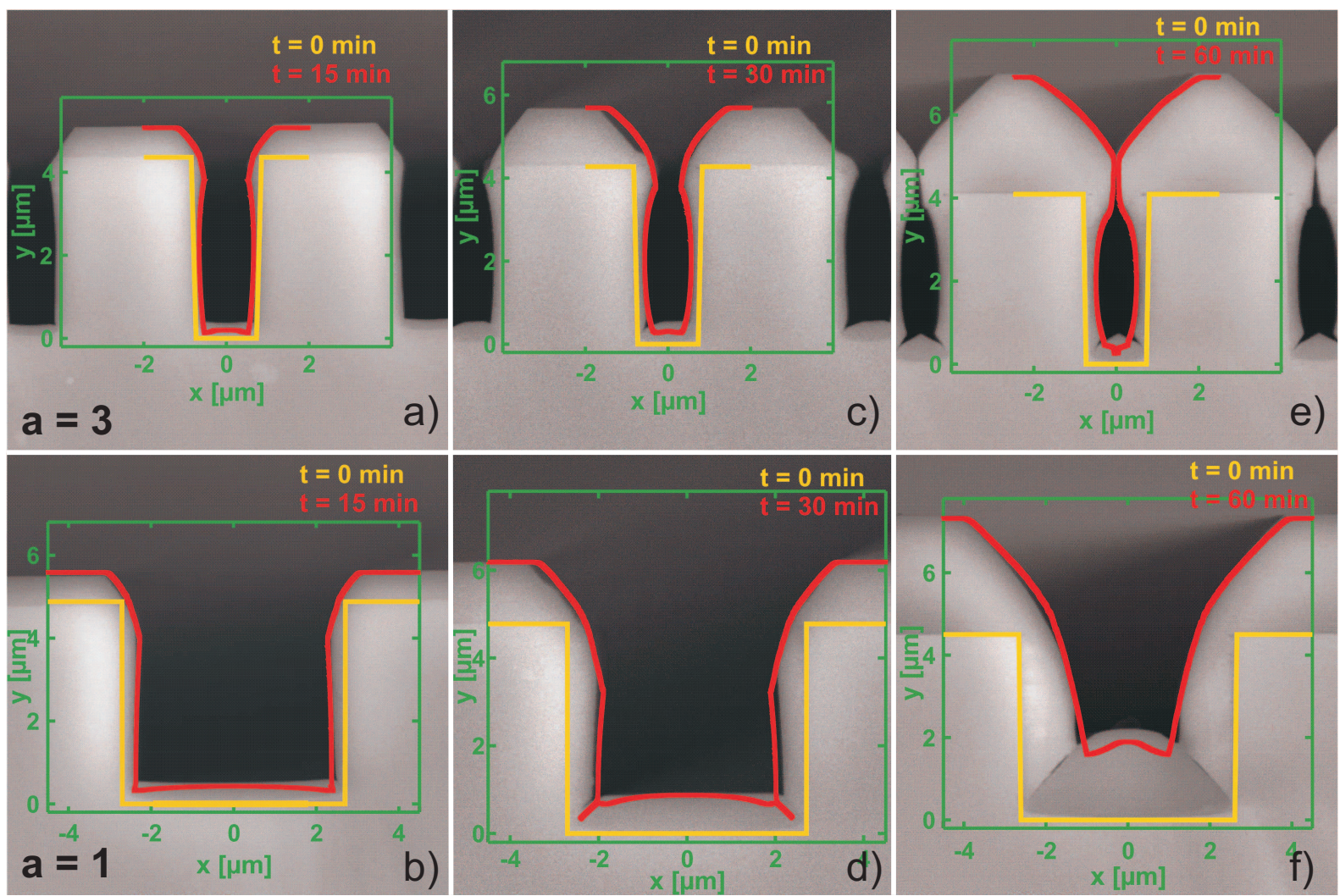

Fig. 4. Comparison of simulated and experimental coatings deposited with substrate bias in the same representation as in Fig. 3 for the same aspect ratios. The reflection of incoming particles at the coating surface turns out to be zero at all time steps.

the coating are reproduced very well. A difference between simulation and experiment is observed at the bottom of the cavities. This can be seen best after 60 min deposition in Figs. 4e,f, where a smaller layer thickness is produced by the simulations compared with the experimental results. Consequently, an additional mechanism, which is not accounted for in the code, must contribute to the layer growth. A possible mechanism is the entrainment of material, which is sputtered or reflected at the edge by the incoming flux of the high-energetic ions into the cavity. This is a process, which occurs in the plasma phase and is not considered in the code SDTrimSP-2D, which only computes the interaction of the particles from the plasma with the substrate atoms. 


\section{$6 \quad$ Film-forming processes}

In a common PECVD process, the shape of the deposited layer can be explained mainly by the geometry of the substrate surface (shadowing effects), the angular distribution of the incoming particles and their sticking probability. Thus, the layer growth of these coatings can be simulated with the already existing Monte-Carlo codes, which can be found in literature [10-14]. In such a deposition process, the chemical reaction of the layer-forming radicals with the target or the already grown coating is more or less insignificant, because of the (low) thermal energy of the film-forming radicals. Hence, the mechanism, which leads to the layer growth and, therefore, to the observed shape of the coating, is only the adherence of the incoming radicals.

By applying a bias voltage to the substrate, the ions are accelerated from the plasma to the substrate surface. Their high-energetic impinge on the surface leads to an increase of the total surface mobility of all target atoms. Thus, the interaction of the impinging ions and the target (collision cascades inside the target) becomes important for the layer growth. In addition to the local deposition, the code SDTrimSP-2D calculates the local reflection, loss, sputtering and redeposition. The locally lost atoms have the opportunity to redeposit at another position with the sticking probability $s$. Figure 5 explains the representations of these values in dependence of the normalized coating surface. This example shows the case for a deposition time of 0 min, i.e. the beginning of the deposition. To this end, the surface is expanded and then replicated on the abscissa. The positions $t_{1}$ and $t_{2}$ represent the edges on the top of the flanks, $b_{1}$ and $b_{2}$ the corners at the bottom of the cavity. The following diagrams are depicted in this way.

In Figs. 6a and b, the local reflection, loss, sputtering and deposition calculated with regard to the ions and the radicals are shown. In Figs. 6c and d, these parameters are opposed to those analyzed on the basis of the ion influence only. In Figs. 6a and b, the local deposition exceeds the local loss. Otherwise, no coating would be deposited and a sputter process would be obtained. The local deposition has its largest value on the upper surface of this geometry and inside the cavity it is reduced, especially at the flanks. In order to 


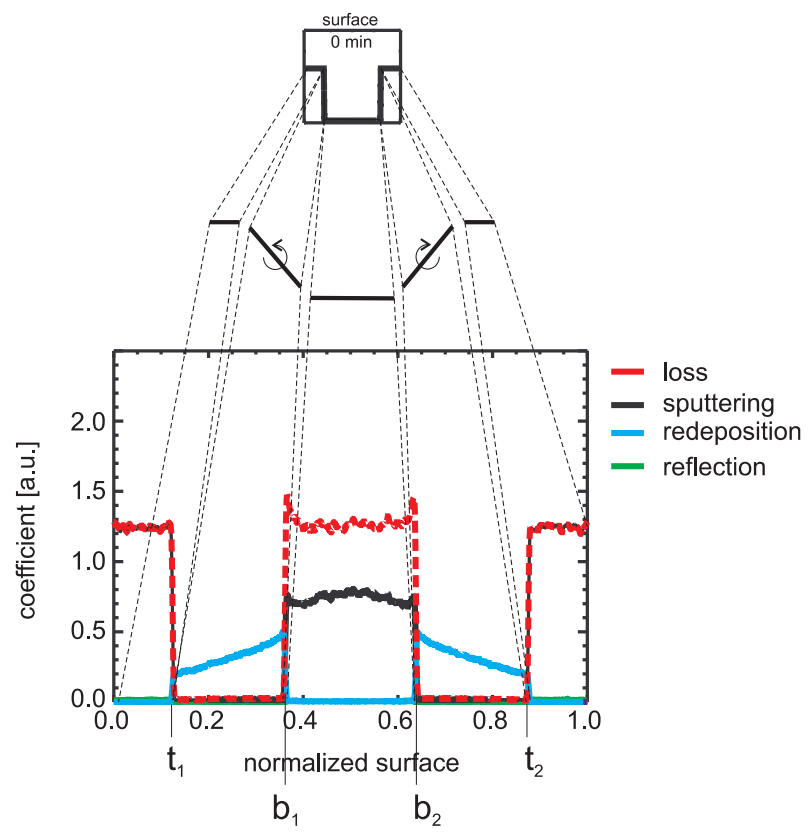

Fig. 5. Illustration of the normalized surface as abscissa for representing the code output.

distinguish between directly deposited particles (mainly radicals) and redeposited ones (mainly induced by the ions), the same parameters are depicted in Figs. 6c and d for the case, where the effect of the ions is taken into account. Obviously, the local loss, sputtering and reflection are affected only by the ions, because they show the same characteristics as in Fig. 6a and b. However, the characteristics as well as the values of the local deposition changes significantly, which is now determined by the redeposition of lost particles. In order to understand the differences in the layers formed with and without substrate bias, the effect of the impinging ions can now be isolated. The influence of only the ions will be examined in the following.

Figure 7 shows the local loss, sputtering, redeposition and reflection in dependence of the normalized film surface at three different time steps of the deposition process $(0,30$ and $60 \mathrm{~min}$ ) and for the coating on the two cavity dimensions ( $a=1$ and $a=3$ ). The deposition time of 0 min (Figs. 7a and b) represents the geometry of the uncoated model substrate. At the beginning of the deposition, the main occurring process is the local loss on the horizontal surfaces on the top of the substrate as well as at the bottom of the trench. Because of the vertical incident of the ions, a particle loss on the parallel flanks cannot occur. The atoms lost at the top of the substrate are sputtered; they leave the 

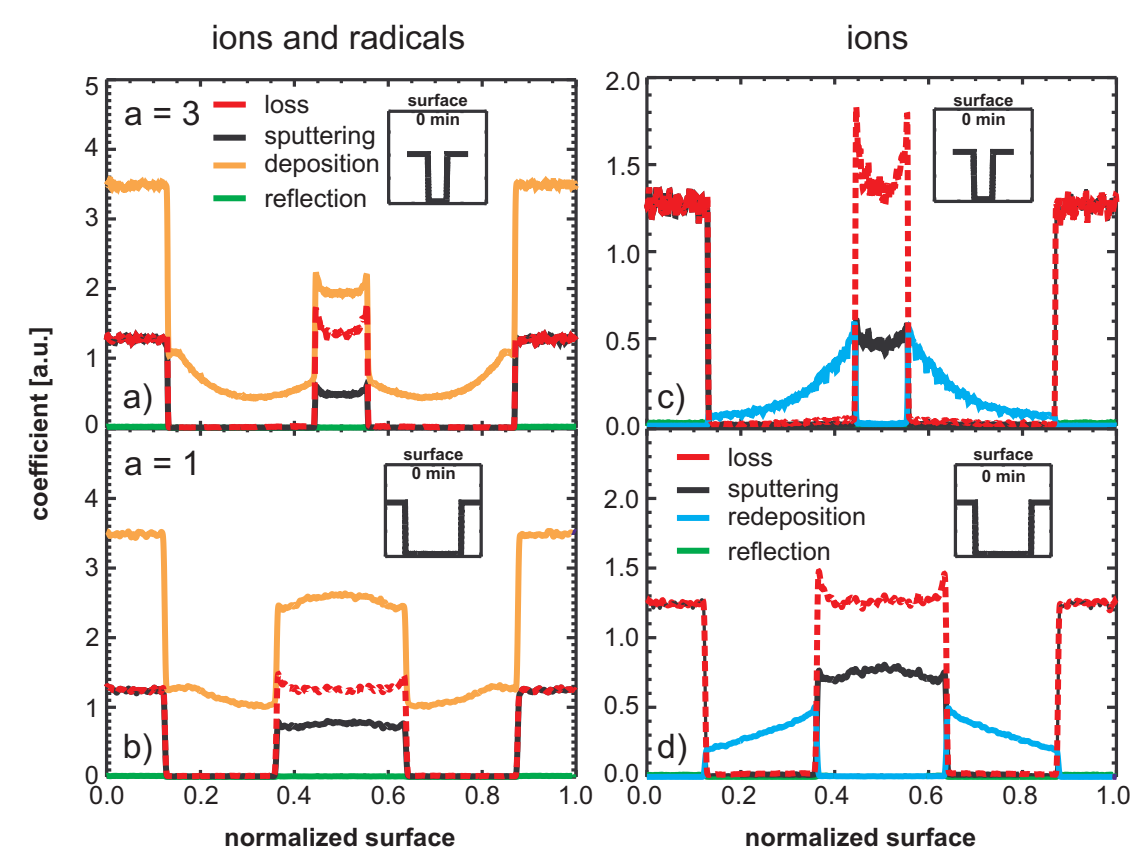

Fig. 6. Local layer-forming mechanisms (reflection, loss, deposition and sputtering) at the beginning of the deposition on a trench with an aspect ratio of $a=3(\mathrm{a}, \mathrm{c})$ and $a=1$ (b,d). The local processes are depicted in dependence of the normalized film surface. a,b) Influence of radicals and ions on the parameters, c,d) ion influence on these parameters, only.

substrate surface since redeposition on the horizontal surface is not possible. The lost particles at the bottom of the cavity can leave it or be redeposited at the flanks of the indentation. The greatest value of the redeposition occurs at the lower edges of the trench $b_{1}$ and $b_{2}$ and decreases upwards. This explains the observed coating at the flanks. The number of the lost particles is the sum of the sputtered and redeposited ones.

Another peculiarity are the two peaks occurring in the local loss and sputter yields. These peaks correspond to the bottom corners $b_{1}$ and $b_{2}$, which do not have any neighbor cells on one side. Hence, one half of the compensating particle flux is missing. The consequence is a higher loss of particles. Additionally, this is intensified by geometrical focussing effects of the particles in these corners. These peaks are observed in [26], too, where the sputtering of a surface structure was analyzed.

Figures $7 \mathrm{~b}$ and e show the situation after a deposition time of $30 \mathrm{~min}$ for the two considered trenches. A distinct feature is the change at the flattened layer edges $t_{1}$ and $t_{2}$. The local loss is clearly increased at these positions compared to the one on the horizontal surfaces. 


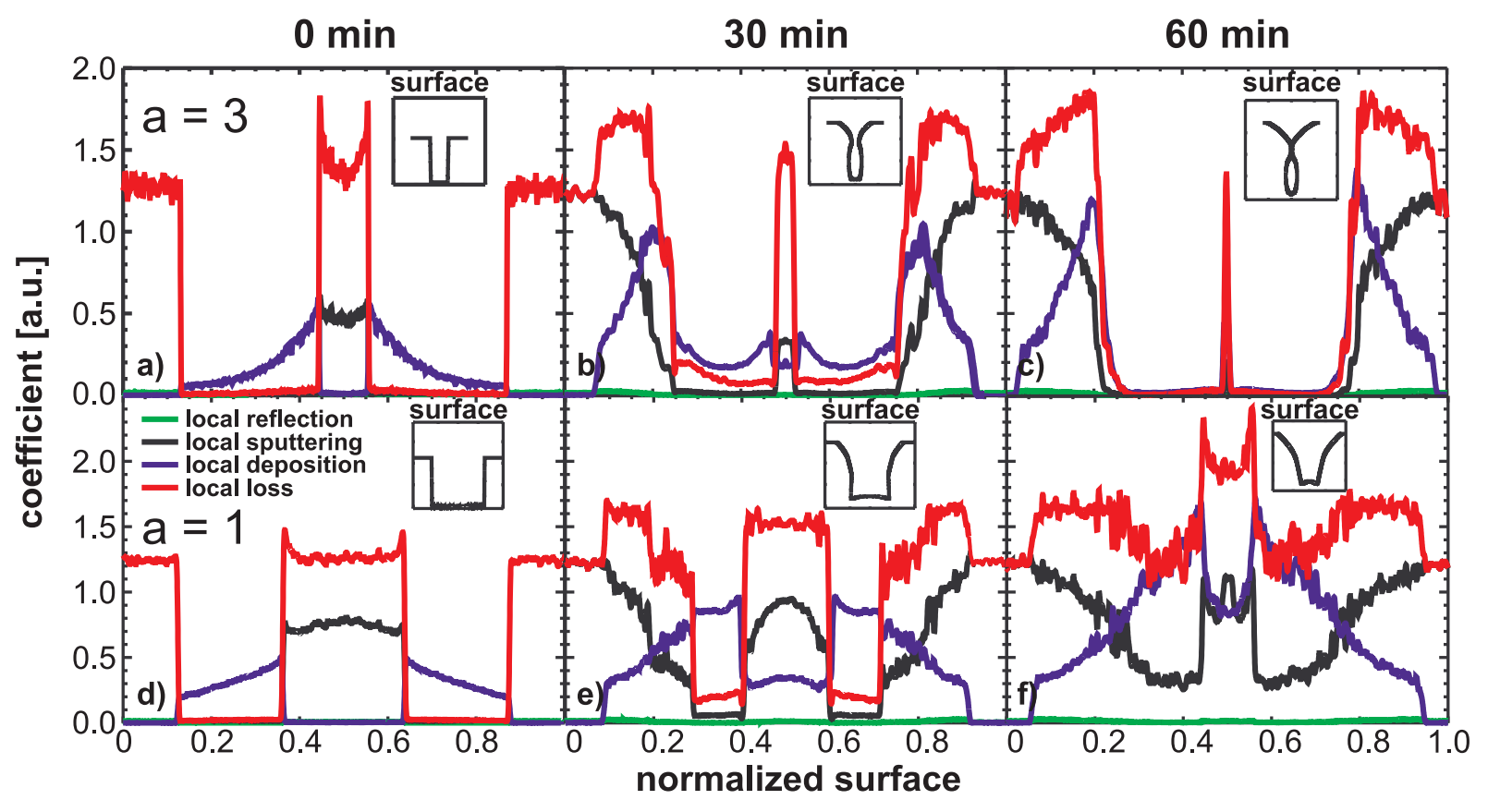

Fig. 7. Local layer-forming mechanisms (reflection, loss, redeposition and sputtering, induced only by ion bombardment) at different deposition times (0,30 and $60 \mathrm{~min}$ ) on a trench with an aspect ratio of $3(\mathrm{a}, \mathrm{c}, \mathrm{e})$ and $1(\mathrm{~b}, \mathrm{~d}, \mathrm{f})$. The local processes are depicted in dependence of the normalized coating surface.

The maximum value of this parameter is found at an angle of incidence of $45^{\circ}$ of the ions on the film surface. Like for the situation at $t=0 \mathrm{~min}$, the number of lost particles on the horizontal surface at the outside of the indentation is governed by the sputtered ones. The situation inside the cavity is also comparable with the initial situation: The atoms lost at the bottom can leave the film surface, or they can be redeposited. For the redeposition, a clear difference to the first case can be observed: The particles lost at the flattened layer edges above have the opportunity to enter the indentation. To explain the redeposition at the flattened layer edges $t_{1}$ and $t_{2}$ above the cavity, the two different aspect ratios have to be distinguished: At the smaller trench in Fig. 7b, the cavity is almost closed by the deposited film. The layer edges overlap the gap. Hence, the lost particles inside the hole have almost no opportunity to leave the indentation. This first leads to a decreasing and then again increasing redeposition along the upward flanks. At the positions, where the formed layer tends to close the cavity, the local redeposition has its largest value. On the one hand, this is caused by the process inside the cavity as explained. On the other hand, the particles, which are lost at the flattened coating edges 
above the closure, are increasingly prevented from entering the cavity and, therefore, contribute to the redeposition, too. Due to the abrasion of the edges, the closure of the film is drawn deeper inside the trench compared to the unbiased case. In comparison, the wider cavity depicted in Fig. 7d shows a smooth hill-shaped characteristics of the redeposition at the bottom of the trench and an almost constant value of this parameter alongside the flanks. The sputter characteristics is similar to the one of the small trench. Most of the sputtered atoms are lost in the middle of the bottom level, without colliding with the film surface and then being redeposited.

At $t=60 \mathrm{~min}$, the smaller trench (Fig. 7c) is narrowed further. Hence, only in the very middle of the cavity, particles can be lost. The parameter characteristics outside the indentation is comparable to that in Fig. 7b. For the wider cavity in Fig. 7f, the situation is similar to that in Fig. 7e with the only difference that the flanks are not parallel to the ion direction anymore. They are formed aslope by the lost and redeposited particles. As a consequence, the local loss at the flanks is non-zero.

In general, it is observed that the decrease of the local redeposition results in an increase of the local sputtering.

\section{Conclusions}

For the specific manipulation of the barrier-layer properties, a detailed analysis of the layer-growth mechanisms in microwave-PECVD was carried out for Si-wafers with a definded surface structure in the $\mu m$ range. To be able to compare the experimental results with simulations using the $2 \mathrm{D}$ binary collision code SDTrimSP-2D the deposition of a-Si:H (hydrogenated amorphous silicon) layers in pure monosilane plasmas were used acting as model system, in which chemical reactions can be neglected. The trench structure of the model substrates from the experiment served as "model cavities".

The differences in biased and unbiased coatings were analyzed in more detail and it turned 
out that without bias, the layer tends to close above the cavities from both sides, but cracks remain at the closure positions. By biasing the substrate, however, a smoothing of the layer edges above the cavities occurred. Thus, the cavities remained open for a longer time, and as a consequence, a more homogeneous coating of the notches is obtained. This effect can be explained by the enhanced surface mobility of the layer-forming particles caused by the bombardment of energetic ions as well as by a sputter process induced by the impinge of these ions. Due to the increased energy input, the coating finally merges completely over voids without remaining cracks, which indicates improved barrier properties of the deposited films. This merging was also verified on steel substrates, which are industrially relevant for solar cell production.

The processes, which lead to different profiles of the biased and unbiased layers at positions of indentations on the substrate surface, were determined by detailed analysis of the local layer-forming mechanisms like the loss of particles, sputtering and redeposition at the film surface. These studies were carried out by means of numerical simulations using the Monte-Carlo Code SDTrimSP-2D. Compared to former simulation codes, this one offers the possibility of including the impacting high-energetic ions in the process on the substrate surface and, therewith, indirectly the effect of the substrate bias.

For both, biased and unbiased depositions, simulation and experimental results on the profiles of the coatings were found to be in very good agreement. Hence, the simulation gives reliable information on the layer-growth mechanisms. Furthermore, it could be shown that the particular local growth processes, namely the loss, the sputtering and the redeposition on the film surface, are not affected by the deposited radicals, which only determines the total deposition rate, but by the impinging energetic ions. The radicals strike the substrate only with thermal energy and, hence, they do not contribute to the increased mobility. Their deposition profile is explained by the geometry of the substrate and the angular distribution of the incoming particles. Thus, the impacting ions could be identified to play the key role in the layer-forming process in biased depositions.

The ion impact takes the strongest effect at the upper edge of the flanks where the particle 
loss is highest. A part of the lost particles are redeposited further inside the trench. Therefore, the position of the layer closure above the cavity is drawn deeper inside the trench. The observed increase of the layer thickness inside the notches is mainly caused by redeposition of particles extracted from the top edges of the trench. Thus, the resulting profile form can be traced back to the detailed sputter and redeposition processes. The mechanisms, which lead to the mergence of cracks, are not covered by the code and might rather be due to chemical processes. 


\section{References}

[1] A. Mutzke and R. Schneider. IPP-Report, 12/4, 2009.

[2] GermanPatent. De 197 39894.4-33.

[3] W. Petasch, E. Räuchle, H. Muegge, and K. Muegge. Surf. Coat. Technol., 93:112-118, 1997.

[4] J. Kopecki. Untersuchung zur plasmagestützten Abscheidung von Barriere-schichten für flexible dünnschichtsolarzellen. Diploma thesis, Universität Stuttgart, Stuttgart, 2008.

[5] W. Eckstein, R. Dohmen, A. Mutzke, and R. Scheinder. IPP-Report, 12/3, 2007.

[6] A. Mutzke, R. Schneider, and I. Bizyukov. J. Nucl. Mat., 115:390-391, 2009.

[7] I. Brodie. J. Vac. Sci. Technol., 6(5):795-800, 1969.

[8] I.A. Blech. Thin Solid Films, 6:113-118, 1970.

[9] C.A. Wasik and J.J. Gniewek. J. Vac. Sci. Technol., 8(2):441-445, 1971.

[10] T.C. Tisone and J.B. Bindell. J. Vac. Sci. Technol., 11(1):72-76, 1974.

[11] T.C. Tisone and J.B. Bindell. J. Vac. Sci. Technol., 11(2):519-527, 1974.

[12] J.B. Bindell and T.C. Tisone. Thin Solid Films, 23:31-47, 1974.

[13] R.C. Ross and J.L. Vossen. Appl. Phys. Lett., 45(3):239-240, 1984.

[14] J.G. Shaw and C.C. Tsai. J. Appl. Phys., 64(2):699-701, 1988.

[15] W. Eckstein. Computer Simulation of Ion-Solid Interactions. Springer Verlag, Berlin, 1991.

[16] W. Möller and W. Eckstein. Nucl. Instr. Meth. Phys. Res. B, 2:814-818, 1984.

[17] T. Shimoda, Y. Matsuki, M. Furusawa, T. Aoki, I. Yudasaka, H. Tanaka, H. Iwasawa, D. Wang, M. Miyasaka, and Y. Takeuchi. Nature, 440(6):783-786, 2006.

[18] S.M. Gates, C.M. Greenlief and D.B. Beach. J. Chem. Phys., 93(10):7493-7503, 1990.

[19] J. Zhou, Y. Chen, X. Liu, and S. Zou. Nucl. Instr. Meth. Phys. Res. B, 39:182-184, 1989.

[20] A. von Keudell and J.R. Abelson. Phys. Rev. B, 59(8):5791-5798, 1999. 
[21] A. Matsuda and K. Tanaka. J. Appl. Phys., 60(7):2351-2356, 1986.

[22] A. Matsuda, K. Nomoto, Y. Takeuchi, A. Suzuki, A. Yuuki, and J. Perrin. Surface Science, 227:50-56, 1990.

[23] J. Perrin and T. Broekhuizen. Appl. Phys. Lett., 50(8):433-435, 1987.

[24] W. Bayer. Solar Energy Materials \& Solar Cells, 78:235-267, 2003.

[25] U. Fantz. Spektroskopische Untersuchung und Modellierung von mikrowellenangeregten Silanplasmen. PhD thesis, Universität Stuttgart, Stuttgart, 1995.

[26] I. Bizyukov, A. Mutzke, R. Schneider, and J. Davis. Nuclear Instruments and Methods in Physics Research B, 268:2631-2638, 2010. 


\section{List of Figures}

$1 \quad$ Scheme of Plasmodul 4

2 Voltage characteristics of the applied substrate bias in the monosilane plasma

3 Comparison of simulated and measured layer growth, deposited without substrate bias onto indentations in the substrate surface. The aspect ratio of the two investigated trenches is $a=3$ and $a=1$. The shape of the original substrate cavity is depicted as the line at $t=0 \mathrm{~min}$. The reflection of incoming particles at the coating surface turns out to be zero at all time steps.

4 Comparison of simulated and experimental coatings deposited with substrate bias in the same representation as in Fig. ?? for the same aspect ratios. The reflection of incoming particles at the coating surface turns out to be zero at all time steps.

5 Illustration of the normalized surface as abscissa for representing the code output.

6 Local layer-forming mechanisms (reflection, loss, deposition and sputtering) at the beginning of the deposition on a trench with an aspect ratio of $a=3(\mathrm{a}, \mathrm{c})$ and $a=1(\mathrm{~b}, \mathrm{~d})$. The local processes are depicted in dependence of the normalized film surface. a,b) Influence of radicals and ions on the parameters, c,d) ion influence on these parameters, only. 
7 Local layer-forming mechanisms (reflection, loss, redeposition and sputtering, induced only by ion bombardment) at different deposition times $(0,30$ and $60 \mathrm{~min})$ on a trench with an aspect ratio of $3(\mathrm{a}, \mathrm{c}, \mathrm{e})$ and 1 $(\mathrm{b}, \mathrm{d}, \mathrm{f})$. The local processes are depicted in dependence of the normalized coating surface. 